\title{
Prognostic Significance of Tumor Immunity in Surgically Resected Pulmonary Pleomorphic Carcinoma
}

\author{
KYOICHI KAIRA $^{1,2,3}$, KIMIHIRO SHIMIZU ${ }^{1}$, HIDEKI ENDOH ${ }^{4}$, KAZUYOSHI IMAIZUMI ${ }^{5}$, \\ MITSUHIRO KAMIYOSHIHARA ${ }^{6}$, MASAYUKI SUGANO ${ }^{7}$, OSAMU KAWASHIMA ${ }^{8}$, \\ SHIGEFUMI TANAKA ${ }^{9}$, ATSUSHI FUJITA ${ }^{10}$, HISAO IMAI $^{11}$, YOSHIHITO KOGURE ${ }^{12}$, \\ TETSUNARI OYAMA ${ }^{13}$, TAKAYUKI ASAO ${ }^{14}$ and KEN SHIRABE ${ }^{1,2}$ \\ ${ }^{1}$ Department of General Surgical Science, Gunma University, Graduate School of Medicine, Maebashi, Japan; \\ ${ }^{2}$ Department of Innovative Immune-Oncology Therapeutics, Gunma University, \\ Graduate School of Medicine, Maebashi, Japan; \\ ${ }^{3}$ Department of Respiratory Medicine, Comprehensive Cancer Center, \\ International Medical Center, Saitama Medical University, Saitama, Japan; \\ ${ }^{4}$ Department of Thoracic Surgery, Saku Central Hospital Advanced Care Center, Saku, Japan; \\ ${ }^{5}$ Department of Respiratory Medicine, Fujita Health University, Toyoake, Japan; \\ ${ }^{6}$ Department of General Thoracic Surgery, Japanese Red Cross Maebashi Hospital, Maebashi, Japan; \\ ${ }^{7}$ Department of Respiratory Surgery, Takasaki Medical Center, Takasaki, Japan; \\ ${ }^{8}$ Department of Respiratory Surgery, Shibukawa Medical Center, Shibukawa, Japan; \\ ${ }^{9}$ Department of Respiratory Surgery, Isesaki Municipal Hospital, Isesaki, Japan; \\ ${ }^{10}$ Division of Thoracic Surgery, Gunma Prefectural Cancer Center, Ota, Japan; \\ ${ }^{11}$ Division of Respiratory Medicine, Gunma Prefectural Cancer Center, Ota, Japan; \\ ${ }^{12}$ Department of Respiratory Medicine, Nagoya Medical Center, Nagoya, Japan; \\ ${ }^{13}$ Department of Diagnostic Pathology, Gunma University, Graduate School of Medicine, Maebashi, Japan; \\ ${ }^{14}$ Big Data Center for Integrative Analysis, Gunma University Initiative for Advance Research, Maebashi, Japan
}

\begin{abstract}
Background: Pulmonary pleomorphic carcinoma $(P P C)$ is a rare aggressive neoplasm, with dismal prognosis. Whether tumor immunity is associated with the progressive biological behavior of PPC remains unclear. The purpose of this study was to examine the prognostic significance of tumor immunity-related markers such as programmed death-1 ligand (PD-L1) and $\mathrm{CD}^{+}$or $\mathrm{CD} 8^{+}$tumor-infiltrating lymphocytes (TILs) in patients with surgically resected PPC. Patients and Methods: Ninety-nine patients with surgically resected PPC were assessed by immunohistochemistry. The expression of $P D$ L1, CD4, and CD8 was examined in specimens of the resected tumors. Results: PD-L1 was highly expressed in 61\% (60/99) of lesions and high expression of CD4 and CD8 was identified
\end{abstract}

Correspondence to: Kyoichi Kaira, MD, Ph.D., Department of Respiratory Medicine, Comprehensive Cancer Center, International Medical Center, Saitama Medical University, 1397-1 Yamane, Hidaka-City, Saitama, 350-1298, Japan. Tel: +81 429844111, Fax: +81429844741, e-mail: kkaira1970@yahoo.co.jp

Key Words: Pulmonary pleomorphic carcinoma, tumor immunity, CD4, CD8, PD-L1, prognosis. in $42 \%$ (42/99) and $51 \%$ (51/99) of lesions, respectively. There was no relationship between the expression $P D-L 1$ and the numbers of $\mathrm{CD}^{+}$or $C D 8^{+}$TILs. The expression of PD-L1 was not identified as a significant prognostic marker; however, a low number of $\mathrm{CD}^{+}$TILs was identified as an independent marker for predicting a worse outcome after surgical resection of PPC, especially in patients with an epithelial component of adenocarcinoma or early stage of disease. By univariate analysis, a low number of $C D 8^{+}$TILs was found to be a significant prognostic marker linked to poor overall survival in patients with non-adenocarcinoma components. Conclusion: A low number of $\mathrm{CD}^{+}$TILs is an independent marker for predicting a favorable prognosis after surgical resection in patients with PPC, especially in patients with adenocarcinoma components or early stage of disease.

Pulmonary pleomorphic carcinoma (PPC) is a rare epithelial tumor with aggressive features, and its incidence ranges from 0.1 to $0.4 \%$ of all thoracic cancer (1). The certification of carcinomatous and sarcomatous components for the defined diagnosis of PPC is required; PPC is classified as a subtype of sarcomatoid carcinoma of the lung in the World Health Organization histological classification of lung neoplasms (2, 

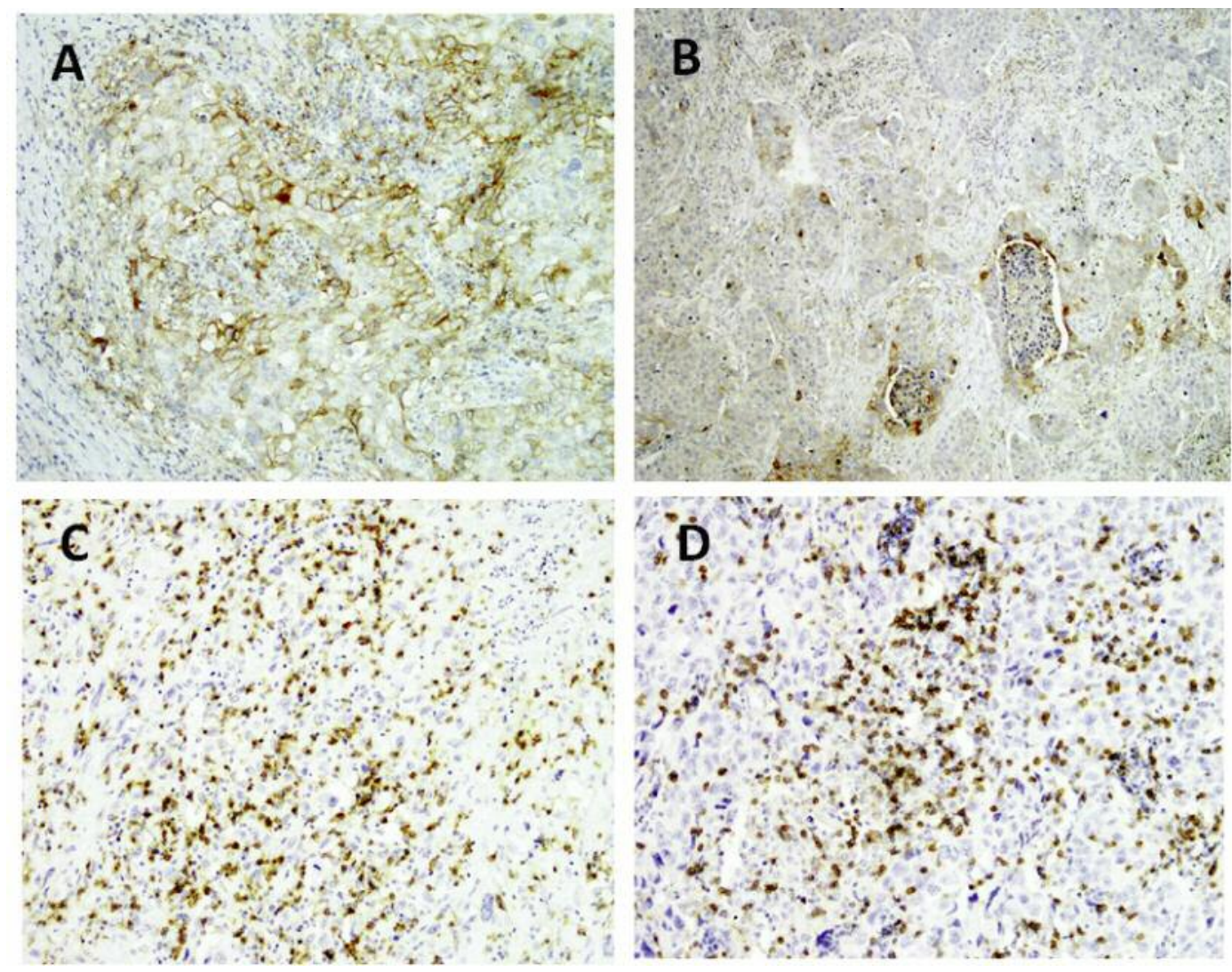

Figure 1. Immunohistochemical staining of programmed death-1 ligand (PD-L1), CD4, and CD8 in patients with pulmonary pleomorphic carcinoma. Immunostaining of PD-L1 shows membranous staining pattern with a score of $6(A)$ and $3(B)$. Representative images of CD4 (C) and CD8 (D) staining for primary tumor-infiltrating lymphocytes are also shown.

3). As PMC has more aggressive malignant potential than other types of thoracic neoplasm, recurrence after surgical resection and poor sensitivity to systemic chemotherapy are recognized as critical clinical issues. Its rarity hampers establishing biomarkers for predicting the outcome of any treatment in patients with PPC. In particular, how tumor immunity suppresses the progressive biological behavior of PPC remains unclear.

Recently, Kim et al. described programmed death-1 ligand (PD-L1) to be highly expressed in 41 patients with PPC and its expression was higher in sarcomatous cells than in carcinomatous areas (4). Although PD-L1 expression has no prognostic implication, a high ratio of programmed death-1 (PD-1) to CD8 tumor-infiltrating lymphocytes (TILs) is suggested to be significantly linked to the shorter progressionfree survival (4). Imanishi et al. reported that PD-L1 expression was positive in $76.9 \%$ of 20 patients with surgically resected PPC and its high expression was closely linked to a favorable overall survival (OS) (5). A recent investigation of pulmonary sarcomatoid carcinomas revealed that PD-L1 expression was higher in sarcomatoid carcinomas than in non-small-cell lung cancer (NSCLC), and a high number of $\mathrm{CD} 4^{+}$TILs constituted a factor for favorable prognosis in survival; however, the expression of PD-L1 was not associated with prognosis (6). Only few studies have discussed the clinicopathological significance of tumor immunity in PPC and the results of these studies are contradictory. Therefore, which tumor immunityrelated markers might predict outcome after treatment in patients with PPC remains unknown. Owing to a small sample size of less than 50 patients in the previous study, drawing optimal prognostic significance of tumor immunity-related markers such as PD-L1 or TILs in PPC proved to be difficult. Indeed, pembrolizumab, an antibody to PD1, reportedly yielded a drastic response in a 71-year-old man with PPC harboring high PD-L1 expression (7). Considering this actual clinical case, how tumor immunity-related markers affect the tumor environment of PPC, including tumor progression, metastasis, and survival, should be elucidated.

Based on these background findings, the aim of our study was to assess the prognostic significance of tumor immunityrelated markers (PD-L1, CD4, and CD8) in patients with surgically resected PPC. 
Table I. Patient demographics according to markers of tumor immunity.

\begin{tabular}{|c|c|c|c|c|c|c|c|c|c|c|c|}
\hline \multirow[b]{2}{*}{ Variable } & \multirow[b]{2}{*}{ Subgroup } & \multirow[b]{2}{*}{$\mathrm{n}=99$} & \multicolumn{3}{|c|}{ PD-L1 (\%) } & \multicolumn{3}{|c|}{ CD4 (\%) } & \multicolumn{3}{|c|}{ CD8 (\%) } \\
\hline & & & $\begin{array}{l}\text { High } \\
(\mathrm{n}=60)\end{array}$ & $\begin{array}{c}\text { Low } \\
(\mathrm{n}=39)\end{array}$ & $p$-Value & $\begin{array}{l}\text { High } \\
(\mathrm{n}=42)\end{array}$ & $\begin{array}{l}\text { Low } \\
(\mathrm{n}=57)\end{array}$ & $p$-Value & $\begin{array}{l}\text { High } \\
(\mathrm{n}=51)\end{array}$ & $\begin{array}{l}\text { Low } \\
(\mathrm{n}=48)\end{array}$ & $p$-Value \\
\hline Age, years & $<69 / \geq 69$ & $51 / 48$ & $28 / 32$ & $23 / 16$ & 0.30 & $19 / 23$ & $32 / 25$ & 0.31 & $31 / 20$ & $20 / 28$ & 0.07 \\
\hline Gender & Male/female & $74 / 25$ & $44 / 16$ & $30 / 9$ & 0.81 & $30 / 12$ & $44 / 13$ & 0.64 & $36 / 15$ & $38 / 10$ & 0.36 \\
\hline Smoking & Yes/no & $80 / 19$ & $48 / 12$ & $32 / 7$ & $>0.99$ & $33 / 9$ & $47 / 10$ & $>0.99$ & $41 / 10$ & $39 / 9$ & $>0.99$ \\
\hline $\mathrm{T}$ Factor & $\mathrm{T} 1,2 / \mathrm{T} 3,4<69 / \geq 69$ & $63 / 36$ & $39 / 21$ & $24 / 15$ & 0.83 & $28 / 14$ & $35 / 22$ & 0.67 & $34 / 17$ & $29 / 19$ & 0.58 \\
\hline Nodal invasion & Yes/no & $41 / 58$ & $32 / 28$ & $26 / 13$ & 0.21 & $13 / 29$ & $28 / 29$ & 0.09 & $14 / 37$ & $27 / 21$ & $<0.01$ \\
\hline Pathological stage & I,II/III,IV & $66 / 33$ & $42 / 18$ & $24 / 15$ & 0.39 & $28 / 14$ & $38 / 19$ & $>0.99$ & $38 / 13$ & $28 / 20$ & 0.09 \\
\hline Lymphatic invasion & Yes/no & $61 / 38$ & $24 / 36$ & $14 / 25$ & 0.83 & $27 / 15$ & $34 / 23$ & 0.68 & $32 / 19$ & $29 / 19$ & 0.83 \\
\hline Vascular invasion & Yes/no & $71 / 28$ & $19 / 41 /$ & $9 / 30$ & 0.49 & $30 / 12$ & $41 / 16$ & $>0.99$ & $38 / 13$ & $33 / 15$ & 0.65 \\
\hline Pleural invasion & Yes/no & $54 / 45$ & $29 / 31$ & $16 / 23$ & 0.53 & $20 / 22$ & $34 / 23$ & 0.31 & $25 / 26$ & $29 / 19$ & 0.31 \\
\hline Adjuvant CTx & Yes/no & $27 / 72$ & $41 / 19$ & $31 / 8$ & 0.25 & $13 / 29$ & $14 / 43$ & 0.50 & $18 / 33$ & $9 / 39$ & 0.07 \\
\hline PD-L1 & High/low & $60 / 39$ & - & - & - & $26 / 16$ & $34 / 23$ & 0.83 & $31 / 20$ & $29 / 19$ & $>0.99$ \\
\hline $\mathrm{CD} 4$ & High/low & $42 / 57$ & $26 / 23$ & $16 / 34$ & 0.83 & - & - & - & $26 / 32$ & $16 / 25$ & 0.10 \\
\hline CD8 & High/low & $51 / 48$ & $31 / 29$ & $20 / 19$ & 0.43 & $26 / 16$ & $25 / 32$ & 0.10 & - & - & - \\
\hline
\end{tabular}

CTx: Chemotherapy; PD-L1: programmed death-1 ligand. Student's t-test was used for continuous variables and chi-squared test for categorical variables. Significant values are shown in bold.

\section{Patients and Methods}

Patients. A total of 105 Japanese patients with histologically defined PPC who underwent surgical resection at multiple institutions between August 2001 and October 2015 were enrolled in this study. Pleomorphic carcinoma was diagnosed according to the 2015 World Health Organization Classification of Tumors (2). Diagnoses were confirmed by light microscopy and immunohistochemistry. PPC was defined as NSCLC containing at least $10 \%$ sarcomatoid components. Out of 105 patients with surgically resected primary tumors, six were excluded because of inadequate tumor specimens for immunohistochemistry. Therefore, 99 patients were eligible for our study in accordance with institutional guidelines and the Declaration of Helsinki at Gunma University Hospital. The Institutional Review Boards of all participating institutions approved this study. The collected tumor samples used in the current study were overlapping with those of our previous study $(8,9)$.

Immunohistochemical staining. For PD-L1, immunohistochemical staining was performed according to the procedures described previously $(10,11)$. Rabbit monoclonal antibody against PD-L1 (E1L3N) (clone E1L3N; 1:200 dilution; Cell Signaling Technology, Danvers, MA, USA) was used. Antigen retrieval was performed by autoclaving using Target Retrieval Solution (AR6, 10× Universal HIER antigen retrieval reagent; Abcam, Tokyo, Japan) and the reaction was visualized using SignalStain Boost IHC Detection Reagent. The expression of PD-L1 was considered positive when membranous staining was observed. A semi-quantitative scoring method was used for PD-L1: $1=<1 \%, 2=1-5 \%, 3=6-10 \%, 4=11$ $25 \%, 5=26-50 \%$, and $6=>50 \%$ of positively stained cells. Tumors with a score $\geq 3$ were graded as having high expression.

Immunohistochemical staining was also performed to detect $\mathrm{CD}^{+}$(1:200 dilution; Dako, Tokyo, Japan) and CD8 ${ }^{+}(1: 1000$ dilution; Abcam) cells in the tumor specimens. After evaluating the specimens, the number of $\mathrm{CD}^{+}$and $\mathrm{CD}^{+}$cells were counted in selected hotspots, using light microscopy under $400 \times$ magnification (field area of $0.26 \mathrm{~mm}^{2}$ ).

We selected the median number of $\mathrm{CD}^{+}{ }^{+}$and $\mathrm{CD} 8^{+}$cells as the cutoff point for $\mathrm{CD}^{+}{ }^{+}$and $\mathrm{CD} 8{ }^{+} \mathrm{TIL}$ density, and patients with fewer than median numbers of these TILs were considered to have low expression. TIL density was estimated for each tumor specimen and infiltration within the tumor and the invasive margins was evaluated. The tissue sections were examined in a blinded fashion by at least two investigators. In cases of discrepancy, both investigators evaluated the slides simultaneously until they reached a final consensus on the assessment. The investigators were blinded to the patients' outcomes.

Statistical analysis. Statistical analyses were performed using Student's $t$ - and chi-squared tests for continuous and categorical variables, respectively. Correlations were analyzed using nonparametric Spearman's rank tests. The Kaplan-Meier method was used to estimate survival as a function of time and survival differences were analyzed by log-rank tests. Overall survival (OS) was the time from tumor resection to death due to any cause. Diseasefree survival (DFS) was the time between tumor resection and the first episode of disease progression or death. Univariate and multivariate survival analyses were performed using the Cox proportional hazards model and a logistic regression model for radical surgery. All $p$-values less than 0.05 were considered statistically significant. All statistical analyses were performed using GraphPad Prism version 4 (GraphPad Software, San Diego, CA, USA) and JMP Pro version 12.0 (SAS Institute Inc., Cary, NC, USA).

\section{Results}

Patient demographics. A total of 99 patients $\left(\mathrm{n}_{\text {males }}=74\right.$, $\mathrm{n}_{\text {females }}=25$; median age $=69$ years; age range $=35-88$ years) were registered in the study. Eighty patients $(81 \%)$ had a 
Table II. Univariate and multivariate analysis of overall and disease-free survival.

\begin{tabular}{|c|c|c|c|c|c|c|}
\hline \multirow[b]{2}{*}{ Variable } & \multirow[b]{2}{*}{ Subgroup } & \multirow[b]{2}{*}{ At 1 year $(\%)$} & \multicolumn{2}{|c|}{ Univariate analysis } & \multicolumn{2}{|c|}{ Multivariate analysis } \\
\hline & & & $p$-Value & HR & $95 \% \mathrm{CI}$ & $p$-Value \\
\hline \multicolumn{7}{|l|}{ Overall survival } \\
\hline Age, years & $<69 / \geq 69$ & $46 / 72$ & 0.48 & & & \\
\hline Gender & Male/female & $61 / 58$ & 0.82 & & & \\
\hline Pathological stage & I,II/III,IV & $73 / 31$ & $<0.01$ & 1.72 & $1.30-2.27$ & $<0.01$ \\
\hline Epithelial type & AC/non-AC & $68 / 49$ & 0.14 & & & \\
\hline Lymphatic invasion & Yes/no & $53 / 71$ & 0.28 & & & \\
\hline Vascular invasion & Yes/no & $58 / 61$ & 0.35 & & & \\
\hline Pleural invasion & Yes/no & $54 / 66$ & 0.09 & & & \\
\hline Adjuvant CTx & Yes/no & $65 / 56$ & 0.14 & & & \\
\hline PD-L1 & High/low & $67 / 49$ & 0.17 & & & \\
\hline CD4 & High/low & $72 / 49$ & 0.03 & 0.77 & $0.58-1.01$ & 0.05 \\
\hline CD8 & High/low & $67 / 50$ & 0.01 & 0.79 & $0.59-1.03$ & 0.08 \\
\hline \multicolumn{7}{|l|}{ Disease-free survival } \\
\hline Age, years & $<69 / \geq 69$ & $41 / 68$ & 0.14 & & & \\
\hline Gender & Male/female & $57 / 46$ & 0.29 & & & \\
\hline Pathological stage & I,II/III,IV & $66 / 33$ & $<0.01$ & 1.66 & $1.26-2.16$ & $<0.01$ \\
\hline Epithelial type & AC/non-AC & $63 / 44$ & 0.56 & & & \\
\hline Lymphatic invasion & Yes/no & $47 / 68$ & 0.68 & & & \\
\hline Vascular invasion & Yes/no & $54 / 52$ & 0.13 & & & \\
\hline Pleural invasion & Yes/no & $43 / 69$ & $<0.01$ & 1.35 & $1.03-1.80$ & 0.02 \\
\hline Adjuvant CTx & Yes/no & $54 / 56$ & 0.83 & & & \\
\hline PD-L1 & High/low & $59 / 42$ & 0.23 & & & \\
\hline $\mathrm{CD} 4$ & High/low & $67 / 44$ & $<0.01$ & 0.72 & $0.54-0.96$ & 0.02 \\
\hline $\mathrm{CD} 8$ & High/low & $56 / 53$ & 0.47 & & & \\
\hline
\end{tabular}

AC: Adenocarcinoma; CI: confidence interval; CTx: chemotherapy; HR: hazard ratio; PD-L1: programmed death-1 ligand. Significant values are shown in bold.

smoking history. Thirty-two patients $(32 \%)$ were diagnosed with stage I, $34(35 \%)$ with stage II, $26(26 \%)$ with stage III, and seven $(7 \%)$ with stage IV PPC. For the histological finding of sarcomatous components, 67 patients had spindle cell type, 10 giant cell type, 10 both spindle cell and giant cell type, and 13 other types. The carcinomatous components of 73 patients included 47 adenocarcinomas, 12 squamous cell carcinomas, eight adenosquamous cell carcinomas, two poorly differentiated carcinomas, and four large-cell carcinomas. Twenty-six patients were found to have histology with a mix of carcinomatous and sarcomatous components. Postoperative survival was measured from the day of the surgery. The median follow-up period was 476 days (range $=32-4,519$ days).

Tumor immunity and clinical correlation. Immunohistochemical assessment was performed using 99 primary sites of PPC. Figure 1 shows representative images of tumor immunity-related markers PD-L1, CD4, and CD8 in PPC samples. PD-L1 immunostaining was predominantly localized in the plasma membrane of tumor cells. The percentage of patients with high PD-L1 expression was $61 \%$ $(60 / 99)$ and the average score was $3.4 \pm 1.8$. The percentage of PD-L1 lesions with scores of 1, 2, 3, 4, 5, and 6 were $15 \%$ (15/99), 24\% (23/99), 13\% (13/99), 14\% (14/99), 13\% (13/99), and $21 \%(21 / 99)$, respectively. High expression of CD4 and CD8 were identified in $42 \%(42 / 99)$ and $51 \%$ (51/99) of lesions, respectively. Concerning the epithelial phenotype, the percentages of patients with $\mathrm{AC}$ and non-AC components with high PD-L1, CD4, and CD8 expression were $60 \%$ and $61 \%$, respectively, $45 \%$ and $40 \%$, respectively, and $55 \%$ and $48 \%$, respectively, without a significant difference between them.

The patients' demographics according to the expression of PD-L1, CD4, and CD8 are listed in Table I. A low number of $\mathrm{CD}^{+}$TILs was significantly linked to the lymph node metastases, however, there was no significant relationship between the expression of PD-L1 or CD4 and other variables. Spearman's rank test revealed that PD-L1 expression was not significantly correlated with CD4 $(\mathrm{r}=-0.05, p=0.62)$ nor $\mathrm{CD} 8(\mathrm{r}=0.13, p=0.21)$ expression.

Survival analysis according to tumor immunity. The median DFS and OS rates for the whole patient cohort were 443 days and 991 days, respectively. The median DFS and OS rates for patients with $\mathrm{AC}$ components were 522 days and 
Table III. Univariate and multivariate analysis of overall and disease-free survival according to epithelial histological type.

\begin{tabular}{|c|c|c|c|c|c|c|c|c|c|c|c|}
\hline \multirow[b]{3}{*}{ Variable } & \multicolumn{6}{|c|}{$\mathrm{AC}$} & \multicolumn{5}{|c|}{ Non-AC } \\
\hline & \multicolumn{3}{|c|}{ Univariate analysis } & \multicolumn{3}{|c|}{ Multivariate analysis } & \multicolumn{2}{|c|}{ Univariate analysis } & \multicolumn{3}{|c|}{ Multivariate analysis } \\
\hline & & At 1 year $(\%)$ & $p$-Value & HR & $95 \%$ CI & $p$-Value & At 1 year $(\%)$ & $p$-Value & HR & $95 \% \mathrm{CI}$ & $p$-Value \\
\hline \multicolumn{12}{|l|}{ Overall survival } \\
\hline Age, years & $<69 / \geq 69$ & $51 / 86$ & 0.49 & & & & $43 / 57$ & 0.63 & & & \\
\hline Gender & Male/female & $63 / 83$ & 0.88 & & & & $55 / 33$ & 0.55 & & & \\
\hline Pathological stage & I,II/III,IV & $76 / 53$ & 0.01 & 1.61 & $1.05-2.49$ & 0.02 & $71 / 13$ & $<0.01$ & 2.28 & $1.49-3.57$ & $<0.01$ \\
\hline Lymphatic invasion & Yes/no & $67 / 72$ & 0.63 & & & & $36 / 71$ & 0.32 & & & \\
\hline Vascular invasion & Yes/no & $70 / 64$ & 0.71 & & & & $47 / 56$ & 0.48 & & & \\
\hline Pleural invasion & Yes/no & $67 / 68$ & 0.10 & & & & $38 / 64$ & 0.49 & & & \\
\hline Adjuvant CTx & Yes/no & $70 / 68$ & 0.63 & & & & $62 / 43$ & 0.05 & & & \\
\hline PD-L1 & High/low & $72 / 64$ & 0.45 & & & & $62 / 31$ & 0.13 & & & \\
\hline $\mathrm{CD} 4$ & High/low & $79 / 59$ & 0.03 & 0.63 & $0.39-0.97$ & 0.03 & $64 / 44$ & 0.29 & & & \\
\hline CD8 & High/low & $67 / 69$ & 0.24 & & & & $67 / 34$ & 0.04 & 0.79 & $0.54-1.13$ & 0.20 \\
\hline \multicolumn{12}{|c|}{ Disease-free survival } \\
\hline Age, years & $<69 / \geq 69$ & $50 / 86$ & 0.49 & & & & $37 / 52$ & 0.37 & & & \\
\hline Gender & Male/female & $62 / 74$ & 0.84 & & & & $51 / 27$ & 0.15 & & & \\
\hline Pathological stage & I,II/III,IV & $65 / 51$ & 0.07 & 1.26 & $0.86-1.86$ & 0.08 & $61 / 16$ & $<0.01$ & 2.39 & $1.57-3.66$ & $<0.01$ \\
\hline Lymphatic invasion & Yes/no & $59 / 69$ & 0.32 & & & & $35 / 60$ & 0.09 & & & \\
\hline Vascular invasion & Yes/no & $63 / 54$ & 0.44 & & & & $42 / 51$ & 0.19 & & & \\
\hline Pleural invasion & Yes/no & $53 / 75$ & 0.09 & & & & $34 / 61$ & 0.02 & 1.31 & $0.84-2.27$ & 0.09 \\
\hline Adjuvant CTx & Yes/no & $60 / 63$ & 0.52 & & & & $55 / 46$ & 0.36 & & & \\
\hline PD-L1 & High/low & $68 / 62$ & 0.69 & & & & $60 / 24$ & 0.06 & & & \\
\hline $\mathrm{CD} 4$ & High/low & $79 / 53$ & 0.01 & 0.55 & $0.35-0.83$ & $<0.01$ & $53 / 38$ & 0.24 & & & \\
\hline CD8 & High/low & $62 / 62$ & 0.71 & & & & $44 / 45$ & 0.45 & & & \\
\hline
\end{tabular}

AC: Adenocarcinoma; CI: confidence interval; CTx: chemotherapy; HR: hazard ratio; PD-L1: programmed death-1 ligand. Significant values are shown in bold.

1038 days, respectively and for patients with non-AC components were 336 days and 361 days, respectively. Of the 99 patients, 58 died and 58 developed recurrence after initial surgery. Table II shows the univariate and multivariate analyses of data for the whole cohort. Univariate analysis revealed that disease stage, and a low number of $\mathrm{CD} 4^{+}$TILs and $\mathrm{CD}^{+}$TILs were significant prognostic markers for predicting worse OS; significant factors for predicting DFS were advanced stage, pleural involvement, and a low number of $\mathrm{CD}^{+}$TILs (Table II). According to the results of the univariate $\log$-rank test, we screened variables with a cut-off of $p<0.05$. Multivariate analysis confirmed that advanced disease, pleural involvement, and a low number of $\mathrm{CD}^{+}$ TILs were independent prognostic factors for predicting a poor DFS and a significant prognostic marker for OS was advanced disease (Table II). Figure 2 shows the KaplanMeier survival curves for patients with high and low expression of PD-L1, CD4, and CD8.

Next, survival analyses according to epithelial histological types and disease staging were performed. By multivariate analysis, a low number of CD4 ${ }^{+}$TILs was identified as an independent prognostic factor for predicting a worse OS and
DFS in patients with AC components, but not in those with non-AC components (Table III). Univariate analysis revealed that a low number of CD4 ${ }^{+}$TILs was a significant prognostic marker linked to poor OS in patients with non-AC components (Table III). The survival analyses showed that a low number of $\mathrm{CD}^{+}{ }^{+}$TILs was an independent prognostic marker for predicting a poor OS and DFS in patients with stage I or II, but not in those with stage III or IV PPC (Table IV).

\section{Discussion}

This was a clinicopathological study wherein we aimed to assess the prognostic significance of tumor immunity-related markers PD-L1 and TILs in surgically resected PPC. We found that PD-L1 was highly expressed in $61 \%$ of PPC regardless of epithelial histological components, however, its expression was not identified as a significant factor for predicting the prognosis after surgical resection. Surprisingly, $\mathrm{CD}^{+}$TILs have been identified as an independent marker for predicting a worse outcome after surgical resection of PPC, especially in patients with AC components or at an early stage of disease. Similarly, there was no relationship 
A

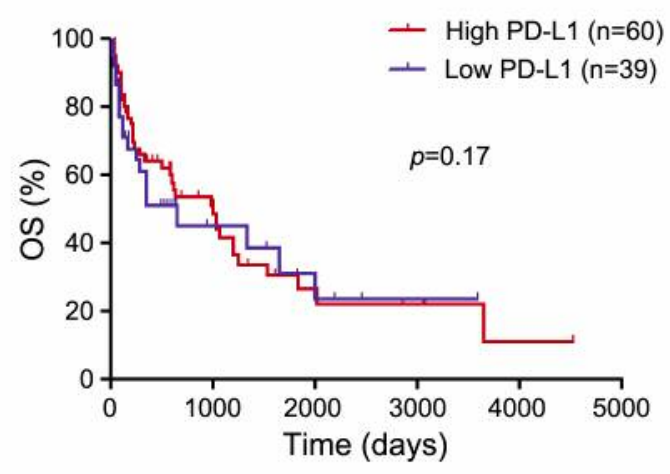

C

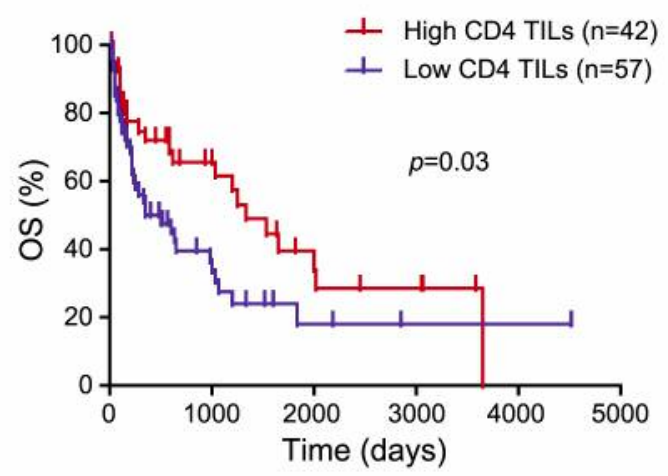

E

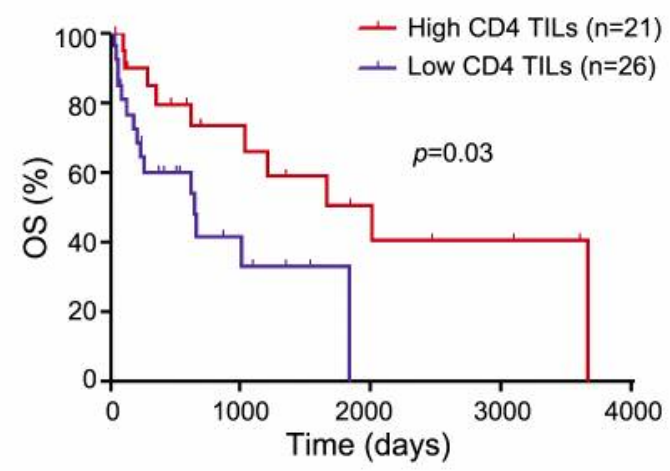

B

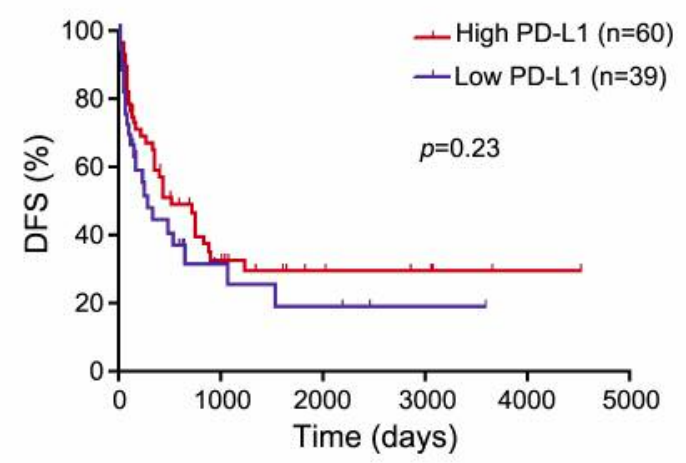

D

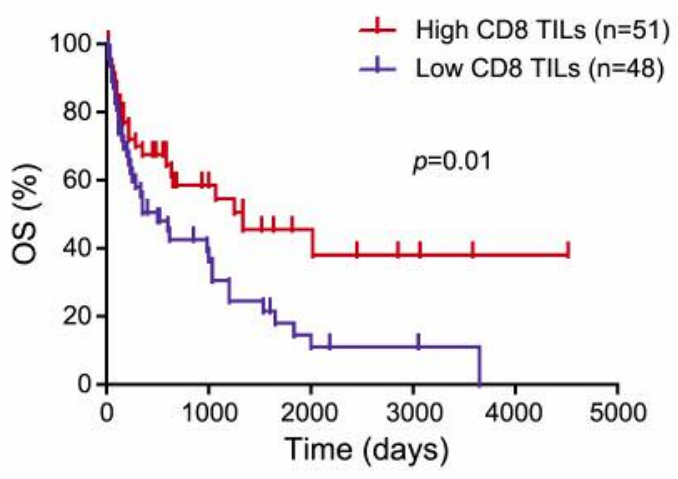

$\mathbf{F}$

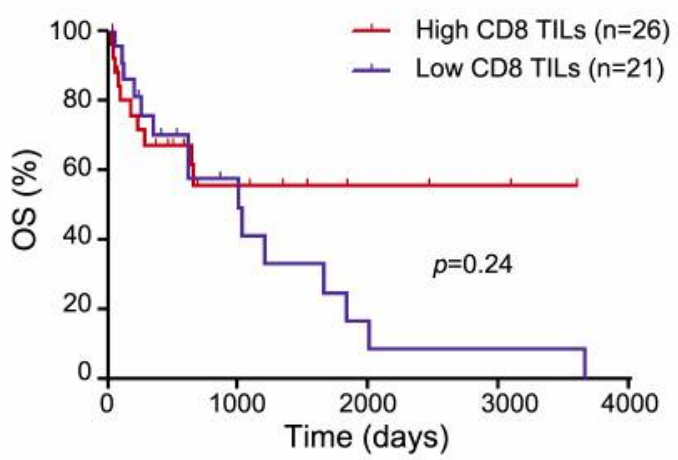

Figure 2. Continued between the expression of PD-L1 and the numbers of $\mathrm{CD} 4^{+}$ or $\mathrm{CD}^{+}$TILs. We analyzed only a limited number of samples from AC and non-AC subpopulations, therefore, a further large-scale study is warranted to elucidate the prognostic significance of TILs in patients with PPC.

Two previous studies have suggested that high expression of PD-L1 was significantly correlated with a worse survival in PPC $(4,12)$, whereas other studies have refuted the prognostic significance of PD-L1 expression $(4,6,12,13)$ and one report supported the notion that PD-L1 expression is closely linked to a favorable survival (5). In this study, we were unable to confirm the prognostic role of PD-L1 expression, regardless of large sample size and use of surgically resected tissues. These studies demonstrated that 
G

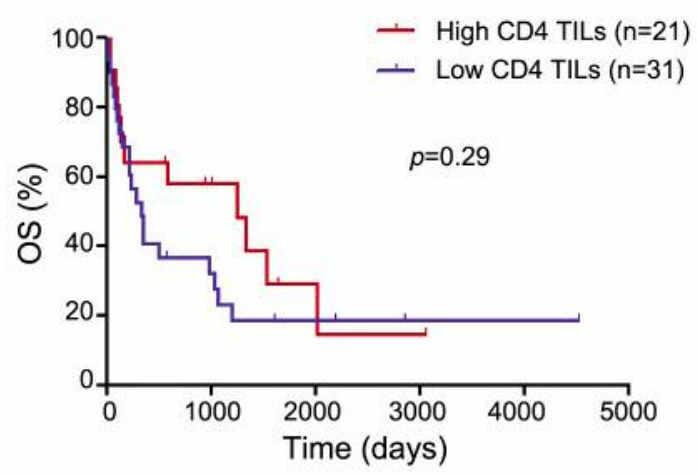

H

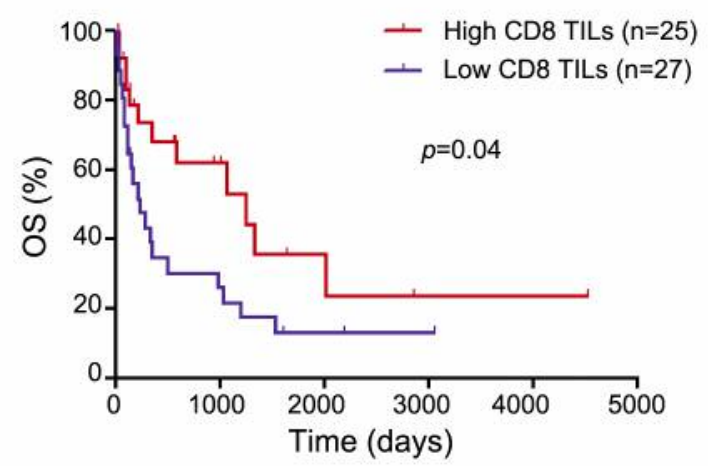

Figure 2. Kaplan-Meier curve of overall survival (OS) (A) and disease-free survival (DFS) (B) according to the expression of programmed death-1 ligand (PD-L1) in the whole patient cohort. No statistically significant difference in the OS and DFS was observed between groups with high and low PD-L1 expression. Kaplan-Meier curves of OS according to the numbers of $\mathrm{CD}^{+}$and $C D 8^{+}$tumor-infiltrating lymphocytes (TILs) revealed a statistically significant difference in the OS between those with high and low CD4 (C) and CD8 (D) expression. Kaplan-Meier survival curve of OS according to epithelial histological type showed a statistically significant difference between those with high and low $C D 4^{+}$TIL numbers in patients with adenocarcinoma component $(A C)(E)$, but not in those with non-AC $(G)$ and $C D 8^{+}$TILs in patients with non-AC component $(H)$, but not in those with $A C(F)$.

PD-L1 is highly expressed in PPC compared to in NSCLC (4, $6,12,13)$; however, the prognostic significance of PD-L1 expression is still debated. Thus, whether PD-L1 expression can predict the outcome after treatment in PPC remains unknown. We speculate that PD-L1 expression may be associated with the pathogenesis and aggressive characteristic of PPC; however, whether it plays a crucial role in predicting prognosis after surgery in PPC remains unclear.

Interferon (IFN) $\gamma$ is produced by $\mathrm{CD}^{+}$and $\mathrm{CD}^{+} \mathrm{T}$-cells and natural killer cells and it has been reported to induce PDL1 expression in tumor cells (14). A recent investigation of 75 patients with sarcomatoid lung carcinomas revealed that $\mathrm{CD}^{+}$ and $\mathrm{CD}^{+}$TILs were identified as significant factor of favorable prognosis (6). Patients with high numbers of $\mathrm{CD}^{+}$ TILs displayed a favorable prognosis when PD-L1 expression was low (6). This report suggests that PD-L1 expression is linked to the exhaustion of lymphocytes and PD-L1 activation may lead to resistance of tumor to TILs rather than an aberrant signaling pathway (6). However, little is known about the relationship between $\mathrm{CD}^{+}$TILs and survival in patients with PPC. Therefore, the results of our study demonstrate that the number of $\mathrm{CD}^{+}$TILs, regardless of the expression level of $\mathrm{PD}-\mathrm{L} 1$, is an important marker and a significant prognostic factor in patients with PPC, especially those having an epithelial component of AC. Similarly, we found that a high number of $\mathrm{CD}^{+}$TILs was also significantly associated with better prognosis after surgery in patients with PPC with nonAC component. In our study, although PD-L1 was highly expressed in PPC similarly to that in previous reports $(4,6,12$, 13), how PD-L1 is linked to the presence of TILs is not yet known. Further in vitro and in vivo studies are warranted to elucidate the prognostic significance of TILs within PPC cells. Okumura et al. reported a case of PPC showing a complete response to anti-PD1 and found that tumor infiltration of $\mathrm{CD}^{+}$ cells was more predominant than that of $\mathrm{CD}^{+}$cells (15); however, the detailed role of the epithelial component is not well understood. In addition to this case report, several cases have been reported to have a good response to anti-PD-1 in PPC $(15,16)$. However, whether the numbers of TILs is able to predict the response to anti-PD1 remains unclear. It is anticipated that anti-PD1/PD-L1 will be an effective regimen for advanced or metastatic PPC.

Recently, Kinoshita et al. described different prognostic values of TILs according to histological types in resected NSCLCs (17). In their study, a high number of CD8 ${ }^{+}$TILs was significantly correlated with a favorable prognosis in patients with non-AC component, but not in those with an $\mathrm{AC}$ component. In addition, a low forkhead box P3/CD4 ratio was identified as favorable prognostic factor in patients with $\mathrm{AC}$ component, suggesting that a high number of $\mathrm{CD} 4^{+}$TILs is associated with better prognosis. Differential analysis according to histology performed in their study corresponds well to that performed in the current study. Therefore, the role of TILs may differ among the epithelial components of PPC.

There are several limitations of the current study. Firstly, the patients registered in our study did not receive antiPD1/PD-L1. Thus, whether PD-L1 is a significant marker for predicting the therapeutic efficacy of PPC by anti-PD1/PDL1 remains unclear. Secondly, it was not possible to analyze the expression of PD-L2 within tumor cells in our cases. Although antibody to PD1 binds to PD-L1 and PD-L2, immunohistochemistry of PD-L2 expression was difficult to 
Table IV. Univariate and multivariate analysis of overall and disease-free survival according to disease staging.

\begin{tabular}{|c|c|c|c|c|c|c|c|c|c|c|c|}
\hline \multirow[b]{3}{*}{ Variable } & & \multicolumn{5}{|c|}{ Stage I or II } & \multicolumn{5}{|c|}{ Stage III or IV } \\
\hline & & \multicolumn{2}{|c|}{ Univariate analysis } & \multicolumn{3}{|c|}{ Multivariate analysis } & \multicolumn{2}{|c|}{ Univariate analysis } & \multicolumn{3}{|c|}{ Multivariate analysis } \\
\hline & & At 1 year $(\%)$ & $p$-Value & HR & $95 \% \mathrm{CI}$ & $p$-Value & At 1 year $(\%)$ & $p$-Value & HR & $95 \% \mathrm{CI}$ & $p$-Value \\
\hline \multicolumn{12}{|l|}{ Overall survival } \\
\hline Age, years & $<69 / \geq 69$ & $59 / 86$ & 0.58 & & & & $24 / 38$ & 0.84 & & & \\
\hline Gender & Male/female & $72 / 76$ & 0.51 & & & & $27 / 36$ & 0.89 & & & \\
\hline Epithelial type & $\mathrm{AC} /$ non-AC & $76 / 71$ & 0.45 & & & & $53 / 13$ & $<0.01$ & 2.15 & $1.35-3.61$ & $<0.01$ \\
\hline Lymphatic invasion & Yes/no & $66 / 80$ & 0.76 & & & & $32 / 20$ & 0.28 & & & \\
\hline Vascular invasion & Yes/no & $65 / 94$ & 0.04 & 1.52 & $1.01-2.43$ & $\mathbf{0 . 0 3}$ & $43 / 11$ & 0.02 & 1.78 & $1.10-2.88$ & 0.01 \\
\hline Pleural invasion & Yes/no & $73 / 76$ & 0.22 & & & & $22 / 41$ & 0.46 & & & \\
\hline Adjuvant CTx & Yes/no & $70 / 75$ & 0.74 & & & & $55 / 19$ & $<0.01$ & 2.31 & $1.38-4.36$ & $<0.01$ \\
\hline PD-L1 & High/low & $81 / 67$ & 0.91 & & & & $31 / 30$ & 0.37 & & & \\
\hline $\mathrm{CD} 4$ & High/low & $88 / 62$ & $\mathbf{0 . 0 3}$ & 0.67 & $0.45-0.96$ & 0.03 & $38 / 24$ & 0.56 & & & \\
\hline CD8 & High/low & $76 / 68$ & 0.11 & & & & $38 / 31$ & 0.45 & & & \\
\hline \multicolumn{12}{|c|}{ Disease free survival } \\
\hline Age, years & $<69 / \geq 69$ & $48 / 82$ & 0.16 & & & & $27 / 45$ & 0.72 & & & \\
\hline Gender & Male/female & $72 / 76$ & 0.51 & & & & $29 / 42$ & 0.60 & & & \\
\hline Epithelial type & $\mathrm{AC} /$ non-AC & $65 / 64$ & 0.87 & & & & $58 / 8$ & $<0.01$ & 2.08 & $1.31-3.39$ & $<0.01$ \\
\hline Lymphatic invasion & Yes/no & $57 / 74$ & 0.41 & & & & $35 / 26$ & 0.71 & & & \\
\hline Vascular invasion & Yes/no & $59 / 75$ & 0.03 & 1.51 & $0.98-2.50$ & 0.05 & $45 / 0$ & 0.06 & & & \\
\hline Pleural invasion & Yes/no & $52 / 76$ & 0.01 & 1.33 & $0.91-2.01$ & 0.13 & $27 / 48$ & 0.30 & & & \\
\hline Adjuvant CTx & Yes/no & $59 / 70$ & 0.56 & & & & $55 / 24$ & $\mathbf{0 . 0 3}$ & 1.83 & $1.17-3.03$ & 0.01 \\
\hline PD-L1 & High/low & $63 / 62$ & 0.94 & & & & $45 / 23$ & 0.32 & & & \\
\hline CD4 & High/low & $75 / 56$ & 0.01 & 0.61 & $0.40-0.88$ & $<0.01$ & $39 / 25$ & 0.66 & & & \\
\hline CD8 & High/low & $76 / 68$ & 0.10 & & & & $33 / 34$ & 0.86 & & & \\
\hline
\end{tabular}

AC: Adenocarcinoma; CI: confidence interval; CTx: chemotherapy; HR: hazard ratio; PD-L1: programmed death-1 ligand. Significant values are shown in bold.

study using commercially available PD-L2 antibody. Finally, our samples were collected from multiple institutions. Therefore, we were unable to validate our results using another cohort, because of the rarity of PPC. A similar study should be performed using a validation cohort.

In conclusion, a low number of $\mathrm{CD}^{+}$TILs is an independent marker for predicting a favorable prognosis after surgical resection in patients with PPC, especially in those with AC components or early-stage disease. PD-L1 was highly expressed in PPC, corresponding to previous reports; however, PD-L1 has prognostic implication. Further study should focus on the relationship between the number of $\mathrm{CD}^{+}$ TILs and the therapeutic efficacy of anti-PD-1/PD-L1 in PPC.

\section{Ethical Approval}

All procedures performed in studies involving human participants were in accordance with the ethical standards of the institutional and/or national research committee.

\section{Conflicts of Interest}

KK has received research grants and a speaker honorarium from Boehringer Ingelheim Company, Ono Pharmaceutical Company, and
Bristol-Myers Company and Chugai Pharmaceutical Company, and KS has received research grants and a speaker honorarium from Ono Pharmaceutical Company and Chugai Pharmaceutical Company. All remaining Authors have declared no conflicts of interest.

\section{Authors' Contributions}

KK and KS designed the study. HE, KI, MK, MS, KO, ST, AF and $\mathrm{HI}$ collected the materials and data. YK, TO and TA performed the statistical analysis. TA and KS reviewed the results, and KK and KS interpreted the data and wrote the manuscript. All Authors read and approved the final version of the paper.

\section{Acknowledgements}

The Authors thank Ms. Sawa Nagayama at Gunma University Hospital for excellent technical assistance.

\section{References}

1 Chang YL, Lee YC, Shih JY and Wu CT: Pulmonary pleomorphic (spindle) cell carcinoma: peculiar clinicopathologic manifestations different from ordinary non-small cell carcinoma. Lung Cancer 4: 91-97, 2001. PMID: 11557118. DOI: 10.1016/ s0169-5002(01)00224-0 
2 Travis WD, Brambilla E, Nicholson AG, Yatabe Y, Austin JHM, Beasley MB, Chirieac LR, Dacic S, Duhig E, Flieder DB, Geisinger K, Hirsch FR, Ishikawa Y, Kerr KM, Noguchi M, Pelosi G, Powell CA, Tsao MS, Wistuba I and WHO Panel: The 2015 World Health Organization Classification of Lung Tumors: Impact of genetic, clinical and radiologic advances since the 2004 classification. J Thorac Oncol 10: 1243-1260, 2015. PMID: 26291008. DOI: $10.1097 /$ JTO.0000000000000630

3 Fishback NF, Travis WD, Moran CA, Guinee DG Jr, McCarthy WF and Koss MN: Pleomorphic (spindle/giant cell) carcinoma of the lung. A clinicopathologic correlation of 78 cases. Cancer 15: 2936-2945, 1994. PMID: 8199991. DOI: 10.1002/10970142(19940615)73:12<2936::aid-cncr2820731210>3.0.co;2-u

4 Kim S, Kim MY, Koh J, Go H, Lee DS, Jeon YK and Chung DH: Programmed death- 1 ligand and 2 are highly expressed in pleomorphic carcinomas of the lung: Comparison of sarcomatous and carcinomatous areas. Eur J Cancer 51: 26982707, 2015. PMID: 26329973. DOI: 10.1016/ j.ejca.2015.08.013

5 Imanishi N, Hirai A, Yoneda K, Shimajiri S, Kuwata T, Tashima Y, Takeuchi M, Iwai Y, Ichiki Y and Tanaka F: Programmed death-ligand 1 (PD-L1) expression in pleomorphic carcinoma of the lung. J Surg Oncol 117: 1563-1569, 2018. PMID: 29601633. DOI: $10.1002 /$ jso.25041

6 Vieira T, Antoine M, Hamard C, Fallet V, Duruisseaux M, Rabbe $\mathrm{N}$, Rodenas A, Cadranel J and Wislez M: Sarcomatoid lung carcinomas shows high levels of programmed death ligand-1 (PD-L1) and strong immune-cell infiltration by CD3 T-cells and marcrophages. Lung Cancer 98: 51-58, 2016. PMID: 27393506. DOI: 10.1016/j.lungcan.2016.05.013

7 Ikematsu Y, Yoneshima Y, Ijichi K, Tanaka K, Harada T, Oda Y, Nakanishi $Y$ and Okamoto I: Marked response to pembrolizumab in a patient with pulmonary pleomorphic carcinoma highly positive for PD-L1. Lung Cancer 112: 230-231, 2017. PMID: 28754417. DOI: 10.1016/j.lungcan.2017.07.020

8 Kaira K, Kamiyoshihara M, Kawashima O, Endoh H, Imaizumi K, Sugano M, Tanaka S, Fujita A, Kogure Y, Shimizu A, Oyama T, Asao T, Shimizu K and Mogi A: Prognostic impact of $\beta 2$ adrenergic receptor expression in surgically resected pulmonary pleomorphic carcinoma. Anticancer Res 39: 395-403, 2019. PMID: 30591486. DOI: 10.21873/anticanres.13125

9 Kaira K, Kawashima O, Endoh H, Imaizumi K, Goto Y, Kamiyoshihara M, Sugano M, Yamamoto R, Osaki T, Tanaka S, Fujita A, Imai H, Kogure Y, Seki Y, Shimizu K, Mogi A, Shitara Y, Oyama T, Kanai Y and Asao T: Author information Expression of amino acid transporter (LAT1 and $4 \mathrm{~F} 2 \mathrm{hc}$ ) in pulmonary pleomorphic carcinoma. Hum Pathol 84: 142-149, 2019. PMID: 30300664. DOI: 10.1016/j.humpath.2018.09.020

10 Kaira K, Shimizu K, Kitahara S, Yajima T, Atsumi J, Kosaka T, Ohtaki Y, Higuchi T, Oyama T, Asao T and Mogi A: 2-Deoxy2-[fluorine-18] fluoro-D-glucose uptake on positron emission tomography is associated with programmed death ligand-1 expression in patients with pulmonary adenocarcinoma. Eur $\mathrm{J}$ Cancer 101: 181-190, 2018. PMID: 30077123. DOI: 10.1016/ j.ejca.2018.06.022
11 Kasahara N, Kaira K, Bao P, Higuchi T, Arisaka Y, ErkhemOchir B, Sunaga N, Ohtaki Y, Yajima T, Kosaka T, Oyama T, Yokobori T, Asao T, Nishiyama M, Tsushima Y, Kuwano H, Shimizu K and Mogi A: Correlation of tumor-related immunity ${ }^{18}$ F-FDG-PET in pulmonary squamous-cell carcinoma. Lung Cancer 119: 71-77, 2018. PMID: 29656756. DOI: 10.1016/ j.lungcan.2018.03.001

12 Chang YL, Yang CY, Lin MW, Wu CT and Yang PC: High coexpression of PD-L1 and HIF-1 $\alpha$ correlates with tumour necrosis in pulmonary pleomorphic carcinoma. Eur J Cancer 60 : 125-135, 2016. PMID: 27107327. DOI: 10.1016/j.ejca. 2016.03.012

13 Yvorel V, Patoir A, Casteillo F, Tissot C, Fournel P, Stachowicz ML, Karpathiou G, Tiffet O, Péoc'h M and Forest F: PD-L1 expression in pleomorphic, spindle cell and giant cell carcinoma of the lung is related to TTF1, p40 expression and might indicate a worse prognosis. PLoS One 12: e0180346, 2017. PMID: 28671973. DOI: 10.1371/journal.pone. 0180346

14 Pardoll DM: The blockade of immune checkpoints in cancer immunotherapy. Nat Rev Cancer 12: 252-264, 2012. PMID: 22437870. DOI: $10.1038 / \mathrm{nrc} 3239$

15 Okamura K, Fukuda Y, Soda H, Ogawara D, Iwasaki K, Fuchi S, Suyama T, Yoshida M, Harada T, Fukuda M and Mukae H: Pulmonary pleomorphic carcinoma with few PD-1-positive immune cells and regulatory T-cells that showed a complete response to nivolumab. Thorac Cancer 9: 193-196, 2018. PMID: 29131510. DOI: $10.1111 / 1759-7714.12557$

16 Kanazu M, Uenami T, Yano Y, Nakatsubo S, Hosono Y, Ishijima M, Akazawa Y, Yamaguchi T, Urasaki K, Mori M and Yokota S: Case series of pleomorphic carcinomas of the lung treated with nivolumab. Thorac Cancer 8: 724-728, 2017. PMID: 28881488. DOI: $10.1111 / 1759-7714.12505$

17 Kinoshita T, Muramatsu R, Fujita T, Nagumo H, Sakurai T, Noji S, Takahata E, Yaguchi T, Tsukamoto N, Kudo-Saito C, Hayashi Y, Kamiyama I, Ohtsuka T, Asamura $\mathrm{H}$ and Kawakami Y: Prognostic value of tumor-infiltrating lymphocytes differs depending on histological type and smoking habit in completely resected non-small-cell lung cancer. Ann Oncol 27: 2117-123, 2016. PMID: 27502728. DOI: 10.1093/annonc/mdw319
Received November 14, 2019

Revised November 24, 2019

Accepted November 28, 2019 